\title{
Insight
}

\section{Regional Farm Diversity Can Reduce Vulnerability of Food Production to Climate Change}

\author{
Pytrik Reidsma $^{1}$ and Frank Ewert ${ }^{1,2}$
}

\begin{abstract}
Food production must adapt in the face of climate change. In Europe, projected vulnerability of food production to climate change is particularly high in Mediterranean regions. Increasing agricultural diversity has been suggested as an adaptation strategy, but empirical evidence is lacking. We analyzed the relationship between regional farm diversity (i.e., diversity among farm types) and the effects of climate variability on regional wheat (Triticum spp.) productivity. An extensive data set with information from more than 50000 farms from 1990 to 2003 was analyzed, along with observed weather data. Our results suggest that the diversity in farm size and intensity, particularly high in Mediterranean regions, reduces vulnerability of regional wheat yields to climate variability. Accordingly, increasing regional farm diversity can be a strategy through which regions in Europe can adapt to unfavorable conditions, such as higher temperatures and associated droughts.
\end{abstract}

Key Words: climate change; farm diversity; regional crop productivity; vulnerability

\section{INTRODUCTION}

Food production is an important ecosystem service that is central to human welfare (Costanza et al. 1997). Climate change will increase risks for food production in large parts of the world (Gitay et al. 2001, Parry et al. 2004). In Europe, food production in Mediterranean regions is projected to be particularly vulnerable to climate change and associated increases in climate variability (Olesen and Bindi 2002, Schröter et al. 2005). This is explained mainly by the negative effects on crop productivity of increasing temperatures and decreasing precipitation.

The extent to which systems are vulnerable to climate change depends on the actual exposure to climate change, their sensitivity, and their adaptive capacity (Intergovernmental Panel on Climate Change (IPCC) 2001). The vulnerability then considers the projected changes in ecosystem services due to climate change compared with the current situation. In contrast to species in natural ecosystems, farmers, assisted by governments, can plan to adapt to climate change (e.g., Smit et al. 2001). Although there is increasing attention to adaptation (IPCC 2007), quantitative understanding of relationships that determine adaptation remains limited. Better understanding of adaptation is needed in order to improve projections of agricultural vulnerability and to prevent or alleviate climate change impacts.

Greater diversity is believed to increase the ability of systems to withstand shocks and thereby decrease vulnerability (Gunderson and Holling 2002). It has been demonstrated that temporal stability of a natural ecosystem increases with increasing species diversity (Díaz and Cabido 2001, Tilman et al. 2006). Also, for agricultural systems, it has been suggested that a greater diversity can decrease vulnerability (e.g., Fraser et al. 2005), but empirical evidence is lacking.

Agricultural diversity can be measured at different levels of organization (farm, region, country, etc.). At the farm level, diversity relates to the diversity in farming activities (e.g., differences in crops grown, fertilizer and biocide use, irrigation). As different crops respond differently to climate variability, greater crop diversity on farms can decrease the vulnerability of farmers' livelihood to climate variability (e.g., Ellis 2000). At the regional level, diversity relates to the diversity among farm 
types (e.g., differences in farm intensity and farm size). Regional farm diversity reflects diversity in management, which largely influences crop productivity (Reidsma et al. 2007).

Although adaptation strategies (e.g., change in sowing date, crop, or cultivar) are mainly adopted at the farm level, in this study we concentrate on the aggregated effects emerging at the regional level, as this is the level at which most impact studies are performed (Bouwman et al. 2006, IPCC 2007, Schröter et al. 2005). We are primarily interested in the vulnerability of regional crop productivity. Associated impacts on the vulnerability of farmers' livelihood are discussed. Accordingly, the objective of this study is to analyze the relationship between regional farm diversity (i.e., diversity among farm types) and the regional effects of climate variability on crop productivity. The analysis is performed using data from an extensive farm survey across the EU15 (i.e., the 15 member states of the European Union before the extension in 2004). Some of the results obtained are additionally supported by a supplementary analysis considering model simulations from a crop growth model (WOFOST; Lazar and Genovese 2004).

\section{METHODS}

\section{Data Description}

Our analysis is based on an extensive data set on farm characteristics and crop yields of individual farms throughout the EU15, provided by the Farm Accountancy Data Network (FADN) (Source: FADN-CCE-DG Agri and LEI; http://ec.europa.eu/ agriculture/rica/index en.cfm). The FADN is the only source of microeconomic data from agricultural holdings in the EU15 that is harmonized, i.e., the book-keeping principles are the same in all countries. Regions are clustered into HARM regions, a harmonized division developed by the Dutch Agricultural Economics Research Institute (LEI). Data were collected from 19902003 in 100 HARM regions with more than 50000 sample farms. These farms are aggregated into farm types based on land use, size, and intensity (Table 1); important farm characteristics that influence farm performance (Andersen et al. 2007, Reidsma et al. 2007). The farm typology is developed in the EU-funded SEAMLESS project (Andersen et al. 2006, van Ittersum et al. 2008). Size classes are based on economic size units (related to standard gross margins), intensity classes on output per hectare, and land-use classes on specialization and land use (e.g., arable/cereal or dairy cattle/temporal grassland). A farm typology offers a tool to synthesize farm management indicators, such as crop yields or fertilizer use. Farms grouped into the same type have a similar farm management (Andersen et al. 2007).

Daily temperature and precipitation data for the study period were obtained from a pan-European weather database, provided by the European Commission's Joint Research Centre (JRC) Monitoring Agriculture through Remote Sensing techniques project (MARS; www.marsop.info). Data are available on a $50 \times 50 \mathrm{~km}$ grid resolution and are averaged per HARM region. Mean temperature (temp) and precipitation (prec) are calculated per region per year for the main growing period between March and August. Also, mean temperature and precipitation for individual months are calculated (i.e., temp $p_{\text {month }}$ and prec $_{\text {month }}$ ). The study period (1990-2003) covers some of the warmest and driest years in the instrumental record of climate. As it is projected that European summers will experience a pronounced increase in the incidence of extreme warm and dry years (Schar et al. 2004), results from this study will be of interest for projections on climate change impacts.

\section{Vulnerability Analysis}

The vulnerability of regional food production to climate change is measured by the regional effects of climate variability on crop productivity. We assume that regions with larger effects of climate variability on crop productivity have a greater vulnerability of food production to climate change and associated climate variability. The analysis considers wheat (Triticum spp.), as it is the most important crop in Europe and is grown in almost all regions. We excluded all regions with less than 10 years of data and with less than $1 \%$ wheat (by area) in the arable area from the analysis.

As yields increase over time due to technological development (Ewert et al. 2005), an analysis of interannual wheat yield variability requires correction for the trend in wheat yield. The anomalies around the trend can be analyzed against climate variables to assess the impact of climate variability. A linear trend is assumed, as was earlier observed for this period (Calderini and Slafer 1998, 
Table 1. Farm typology. Each farm type is characterized by a land use, size, and intensity dimension.

\begin{tabular}{|c|c|c|}
\hline & Dimension and type & Definition \\
\hline \multicolumn{2}{|c|}{ Land use } & (Specialization), Land-use type rule ${ }^{\mathrm{a}}$ \\
\hline 1 & Arable/cereal & $(1+6),<12.5 \%$ fallow and $>=50 \%$ cereals \\
\hline 2 & Arable/fallow & $(1+6),>=12.5 \%$ fallow \\
\hline 3 & Arable/specialized crops & $(1+6),>=25 \%$ of arable land in specialized crops \\
\hline 4 & Arable/others & $(1+6)$, other arable \\
\hline 5 & Dairy cattle/permanent grass & $(4.1),>=50 \%$ grass and $<50 \%$ temporary grass \\
\hline 6 & Dairy cattle/temporary grass & $(4.1),>=50 \%$ grass and $>=50 \%$ temporary grass \\
\hline 7 & Dairy cattle/land independent & (4.1), UAA $=0$ or $\mathrm{LU} / \mathrm{ha}=>5$ \\
\hline 8 & Dairy cattle/others & (4.1), other dairy cattle \\
\hline 9 & Beef and mixed cattle/permanent grass & (4.2 and 4.3$)$, as 5 \\
\hline 10 & Beef and mixed cattle/temporary grass & (4.2 and 4.3$)$, as 6 \\
\hline 11 & Beef and mixed cattle/land independent & (4.2 and 4.3$)$, as 7 \\
\hline 12 & Beef and mixed cattle/others & (4.2 and 4.3), other beef and mixed cattle \\
\hline 13 & Sheep and goats/land independent & $(4.4)$, as 7 \\
\hline 14 & Sheep and goats/others & (4.4), other sheep and goats \\
\hline 15 & Pigs/land independent & $(5.1)$, as 7 \\
\hline 16 & Pigs/others & (5.1), other pigs \\
\hline 17 & Poultry and mixed pigs/poultry & $(5.2)$ \\
\hline 18 & Mixed farms & (7) \\
\hline 19 & Mixed livestock & $(8)$ \\
\hline 20 & Horticulture & (3) \\
\hline 21 & Permanent crops & $(2)$ \\
\hline \multicolumn{3}{|c|}{ Size } \\
\hline 1 & Small scale & $<16 \mathrm{ESU}$ \\
\hline 2 & Medium scale & $>=16 \mathrm{ESU}$ and $<40 \mathrm{ESU}$ \\
\hline 3 & Large scale & $>=40 \mathrm{ESU}$ \\
\hline
\end{tabular}



Intensity
1 Low intensity
2 Medium intensity
3 High intensity

Total output per ha $<€ 500$ euro

Total output per ha $>=€ 500$ and $<€ 3000$

Total output per ha $>=€ 3000$

a The specialization dimension is based on the EU/FADN farm typology (http://ec.europa.eu/comm/agri culture/rica/diffusion en.cfm). Only the most important land-use type rules are described here; the \% of area relates to the utilized agricultural area (uaa). A full description is given in Andersen et al. (2006).

Ewert et al. 2005, Reidsma 2007). We tested for stationarity along the trend; stationarity exists if the mean and variance of the error terms (i.e., the anomalies) are constant. Although not always significant, trends are calculated for all regions. The absolute anomaly from the trend expressed in tons/ ha is used in the present analysis.

Regional effects of interannual climate variability on wheat yields are measured by the Pearson correlation coefficient $(r)$ between wheat yield anomalies from a linear trend and temp [ryield, temp)] and prec [r(yield,prec)]. The start and length of the growing season differ depending on the region, and result in regional differences for the months that are most important for wheat growth. Therefore, we also calculated Pearson correlations between wheat yield anomalies and average temperatures and precipitations for the six individual months from March to August (i.e., 12 correlations per region).

Furthermore, we calculated Pearson correlations between temp and prec and simulated water limited (Ywat) and potential (Ypot) yields from the Crop Growth Monitoring System (based on WOFOST; Lazar and Genovese 2004, Reidsma 2007). These correlations indicate the potential impact of temp and prec on wheat growth, without considering management and adaptation. Comparing these simulations with the results from the farm survey analysis should further clarify the importance of management and adaptation for explaining regional differences in yield responses to climate variability.

As r(yield,prec) is not significant in any of the regions, and calculations based on individual months are similar to $r$ (yield,temp) (results section), further analysis focuses mainly on r(yield, temp), for which results are especially interesting.

\section{Measures of Regional Farm Diversity}

In this paper, regional farm diversity is considered by two measures. The first measure represents the diversity in the yield responses of farm types in a region. The variation in farm type yield variability (SD) indicates, for a region, the variation among farm types in their interannual yield variability. If yields of different farm types increase and decrease in the same years, SD is low; if farm types show different yield responses, SD is large. A low variation suggests a low diversity in management practices, whereas a larger variation suggests greater diversity in management practices. The SD is measured as the standard deviation in the relative yield anomaly per year of all farm types in a region, averaged over the study period (1990-2003) as

$$
S D=\sum_{t=1}^{N} s d\left(Y_{A, 1}, Y_{A 2}, \ldots, Y_{A, f}\right)_{t} / N \text { with } Y A_{1} i=\frac{100 \cdot\left(y_{i t}-\sum_{t=1}^{N} y_{i t} / N\right)}{\sum_{t=1}^{N} y_{i t} / N}
$$

where $s d$ is the standard deviation of relative yield anomalies $\left(Y_{A, i}\right)$ of farm types $i(i=1,2, \ldots \mathrm{f})$ per year $t(t=1,2, \ldots \mathrm{N})$. Yield anomalies per farm type and year are calculated from the actual yield $(y)$ related to the average of the study period. No trend is considered at farm type level as few trends are significant and trends can be distorted by missing years (Reidsma 2007). Relative yield anomalies are 
considered, as absolute yields differ per farm type within a region, which would affect the comparison among farm types.

The second measure, which we refer to as regional farm diversity, demonstrates the diversity in the abundance of different farm types. This measure indicates how diverse the farm types are in a region according to the farm characteristics (land use, size, intensity) used for the farm typology. The regional farm diversity is expressed by land-use diversity, size diversity, and intensity diversity; based on the Shannon-Weaver index (Shannon and Weaver 1949), indicating the number of farm types and evenness of farm types as

$$
H_{d}{ }^{\prime}=\sum_{i=1}^{f_{d}} \frac{W_{i}}{W^{\prime}} \ln \frac{w_{i}}{W_{i}}
$$

where $f_{d}$ is the number of farm types per dimension $d, w_{i}$ is the wheat area of farm type $i$ and $W$ is the total wheat area in a region. $H_{d}$ ' is calculated with the size $\left(f_{d}\right.$ is number of size types), intensity $\left(f_{d}\right.$ is number of intensity types $)$ and land use $\left(f_{d}\right.$ is number of land-use types) types as input. As the number of land-use types (21) is large compared with size (3) and intensity (3) types, the three dimensions are separated.

Regional farm diversity can be based on these three farm characteristics as these factors were identified as having most influence on crop productivity and they synthesize farm management best (Andersen et al. 2007, Reidsma et al. 2007). Although farms can differ in many factors, the farm types clearly differ in management indicators that influence adaptation. For example, high intensive farms have a different management with regard to fertilizer and crop protection use compared with low intensive farms.

\section{Quantifying Effects of Regional Farm Diversity on Vulnerability}

Both types of measures of regional farm diversity have been related against r(yield, temp). Our main objective is to analyze the relationship between regional farm diversity and regional effects of climate variability on crop productivity [i.e., r(yield, temp)]. We are aware that differences in r(yield, temp) can be determined by many factors, such as regional differences in cultivars. By analyzing the variation in $\mathrm{SD}$, we test whether regional differences (e.g., in cultivars) can account for differences in $r$ (yield, temp). When there is a large variation in yield responses of farm types, yield responses are mainly determined by differences among farm types, and not by regional differences. Subsequently, measuring the SD against r(yield, temp) indicates whether this diversity is related to r(yield, temp).

Secondly, using a linear regression model, we tested how regional farm diversity influences r(yield, temp). Regional farm diversity is expressed by diversity in land use, size, and intensity; based on the Shannon-Weaver index (Eq. 2). To account for other effects, the composition (i.e., presence of farm types) and prevailing climate conditions are also included in the model. For temporal stability of natural ecosystems, it has been demonstrated that both composition and diversity are important (Tilman et al. 2007). Also, for agricultural adaptation, the abundance of specific farm types present in a region (e.g., mainly high intensive farms compared with mainly low intensive farms) likely has an effect, independently of the diversity herein. Differences in the presence of farm types largely account for management factors, such as crop protection use (i.e., a high intensive farm uses more pesticides than a low intensive farm). Composition is represented by the presence of different farm types for size (small, medium, and large scale) and intensity (low, medium and high intensive) within a region, measured as the fraction of the total. For composition of land use, we used the fraction of arable land in total agricultural area and the fraction of wheat area in the total arable land. Furthermore, prevailing climatic conditions are included in the regression model and represented by average temp and prec of the whole period. The backward procedure is used to ensure only significant relationships $(\mathrm{p}<0.10)$ are included.

\section{RESULTS}

\section{Effects of Climate Variability on Wheat Yields}

Spatial variability in average wheat yield (from 1990-2003) is significantly negatively correlated to average temp (Fig. 1). Wheat yields are thus lower in regions with higher temperatures. Therefore, it is expected that higher temperatures will generally 
have a negative effect on temporal variability in wheat yields. However, interannual yield variability is affected by temp, but $r$ (yield,temp) varies among regions. Interestingly, $r$ (yield,temp) is significantly negative $(r<-0.53)$ in many temperate regions and low in most Mediterranean regions with typically higher temperatures (Fig. 2). The r(yield,prec) is not significant in any of the regions (Fig. 3).

Results for $r$ (yield,temp) are opposite to what would be expected from simulations with crop models. Effects of higher temperatures are projected to be more negative for potential and water-limited yields (Ywat) in Mediterranean regions compared with temperate regions (Fig. 4). Therefore, we are interested in factors that explain the pattern for $r$ (yield,temp).

Even in many Mediterranean regions, where water limitation is expected to cause more problems for crop growth, yields are only weakly related to the growing season precipitation (Fig. 3). The results for $r$ (yield,temp) are also rarely influenced by the aggregation of temperature variables. Spatial patterns of the effects of temperature of individual months, including the distinct negative effects on yield in temperate regions, are similar to the calculations based on 6-month averages (Fig. 5). It is apparent that in many neighboring temperate regions, the same months account for the most negative (significant) temperature effect, whereas in many neighboring Mediterranean regions, different months account for the most negative (but small) temperature effect. Large and similar effects among neighboring regions suggest that relationships are not just coincidental statistical relationships, but causal effects can be assumed, whereas the opposite is true for small and varying relationships.

\section{Effects of Regional Farm Diversity on Regional Vulnerability}

The analysis on the interannual variability in farm type yields revealed that, in regions where temperature effects on yield are less negative, the diversity in farm type yield variability (SD) is greater (Fig. 6, $p=0.04$ ). Hence, where interannual variability in wheat yields differs more among farm types, the relationship with interannual temperature variability is not evident.

Results from the regression model (Table 2) indicate that diversity in size and intensity reduces the on- average negative effects from higher temperatures on regional wheat yields [r(yield,temp)]. Results are less pronounced but negative for land-use diversity. This is likely related to similar land-use types regarding wheat management (i.e., dairy cattle/ temporary grass and beef and mixed cattle/ temporary grass) being grouped in different landuse types. Clearly, yield responses to temperature differ depending on the farm type and temperature effects on regional yields are less pronounced when farm diversity in a region is high. Regional farm diversity, in size and intensity, represents diversity in management strategies (e.g., cultivar choice, and fertilizer and pesticide use). The diversity in management strategies leads to low regional impacts of climate variability (Fig. 6).

In this study, we are mainly interested in the effects of farm diversity, but farm type composition also has an impact. The r(yield,temp) is less negative in regions where the fraction of small-scale farms is larger, whereas it is more negative where mediumscale farms cover a larger part of the area (Table 2). This is possibly related to small-scale farmers having more flexibility to adapt management practices compared with larger farms. Regions with more small-scale farming also have greater farm diversity, as more farms cover the same area.

Wheat yields on medium intensive farms are less negatively impacted by temp than low and high intensive farms. The larger impacts for low intensive farms can be explained by the low technical ability to adapt practices. For high intensive farms, the larger impact is possibly due to management optimized toward prevailing conditions. Management is aimed at achieving wheat yields close to potential yields. If potential yields are approached and management is not adapted year by year, yield variability will be mainly due to climate variability.

\section{DISCUSSION}

\section{Regional Farm Diversity and Agricultural Vulnerability}

The conclusion that Mediterranean regions are most vulnerable to climate change (Olesen and Bindi 2002, Metzger et al. 2006) needs refinement. Such statements are often derived from simulations with mechanistic crop models that strongly emphasize biophysical factors that determine potential and water-limited yields. However, actual yields are 
Fig. 1. Spatial distribution of average wheat yields ( $\mathrm{t} / \mathrm{ha}$ ), and relationships to average temperature (temp, ${ }^{\circ} \mathrm{C}$ ) from 1990-2003. This figure indicates that, spatially, there is a negative relationship between wheat yields and temperature.

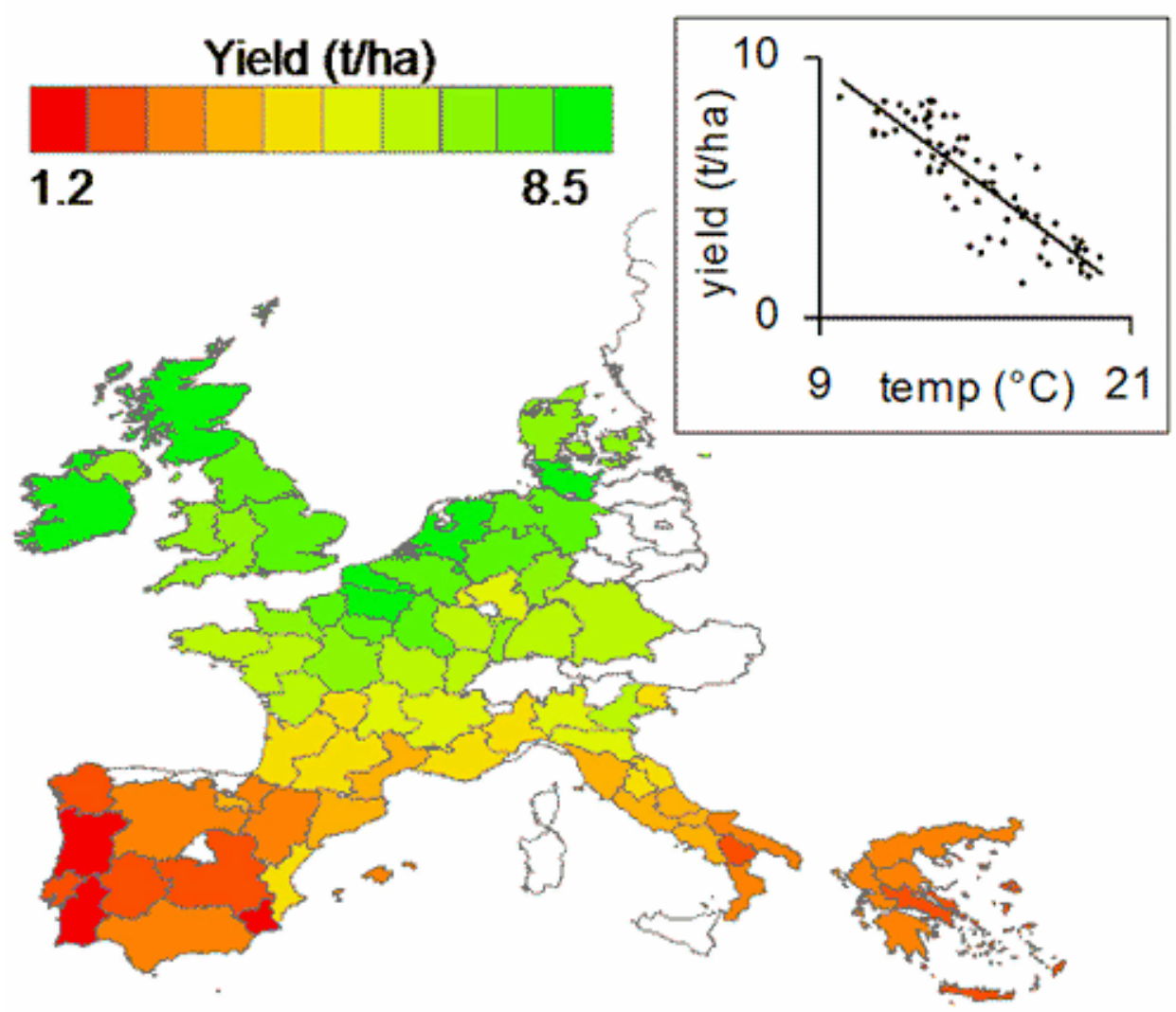

largely influenced by regional socioeconomic conditions and farm management. These factors are often not considered in crop models, but can greatly modify the climate change impacts (Ewert et al. 2007).

The weak relationships between climate variability and wheat yield variability in many Mediterranean regions suggest that farm management here is largely adapted to climate variability. In regions where prevailing climatic conditions are less favorable for wheat growth, farm management is not aimed at achieving maximum yields (Reidsma et al. 2007). It seems more focused on coping with climate variability: as risks are larger, more attention is paid to reducing the impacts of risks. In regions where prevailing climatic conditions are more favorable, farm management is more focused on achieving high yields. Fletcher and Hilbert (2007) showed that the resilience of a system decreases rapidly when maximum profit is approached. When risks are low, aiming for maximizing crop yields (and profit) can be a rational objective, but when risks increase this strategy makes farming systems more vulnerable. In a local study, van der Dries (2002) showed that small-scale traditional farmers who take into account variation in water availability can cope better with climate variability than modern intensive farms, which are more dependent on external resources. There is a trade-off between obtaining high productivity and maintaining resilience.

In this study, we observe that, especially in regions with high wheat yields, the impact of climate variability on wheat yields is high. Hence, the 
Fig. 2. Spatial distribution of the correlation between inter-annual variability in temperature and wheat yield anomalies [r(yield,temp)], and relationships to average temperature (temp, ${ }^{\circ} \mathrm{C}$ ) from 1990-2003. Based on the relationship for spatial variability (Fig. 1), a negative relationship for temporal variability can be expected; this is the case in many temperate regions, but the relationships are often positive in warmer regions.

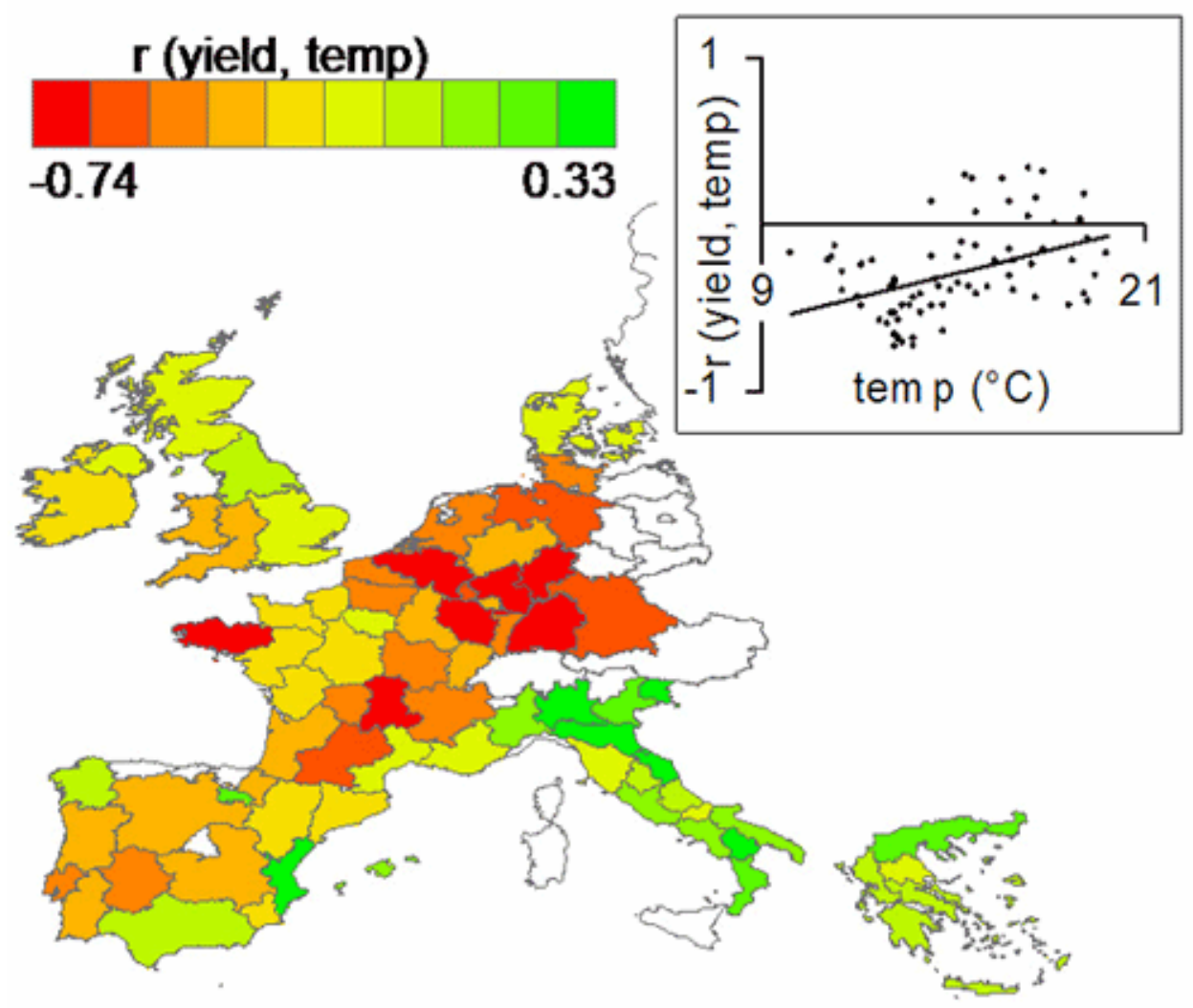

increasing climate variability associated with climate change will mainly decrease the stability of wheat yields in these regions, and adaptation is needed to decrease vulnerability. However, even under climate change, farmers in higher-yielding regions may prefer a higher productivity above a high resilience. The choice for the best strategy depends on farmers' objectives. We did not explicitly analyze how farmers will respond to climate change, but our results suggest that there is less diversity in farming strategies (which is mainly yield maximization) in higher-yielding regions than in the lower-yielding Mediterranean regions. Considering such results in impact assessment studies will be required to improve estimates of crop responses to climate change that are presently reported to be most negative for Mediterranean regions (Olesen and Bindi 2002, Easterling et al. 2007).

Other studies mention hazard exposure as being an important indicator for successful agricultural adaptation (e.g., Downing et al. 2001, Smit and Skinner 2002). This seems valid for regions regularly exposed to high temperatures. When risks of higher temperatures and associated droughts are higher, farms need to adapt their management in order to cope with this. Apparently, farms tend to find different ways to manage climatic variability, leading to regional farm diversification. Regional farm diversity reduces vulnerability at the regional level, as the variety of responses at farm level lead 
Fig. 3. Spatial distribution of the correlation between inter-annual variability in precipitation and wheat yield anomalies [r(yield,prec)]. The legend is different from Fig. 2 to demonstrate the (non-) significant relationships $(|\mathrm{r}|<0.53)$.

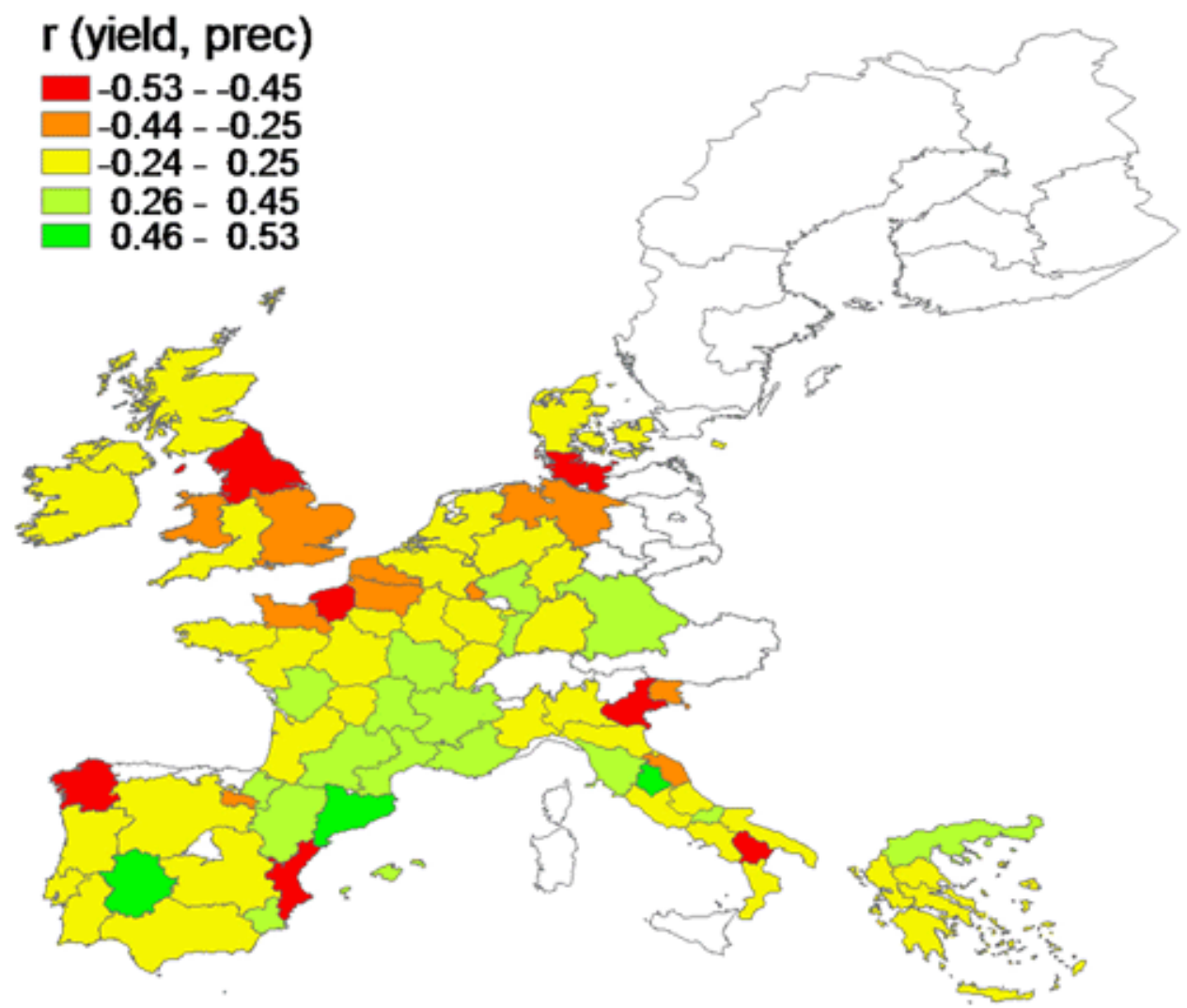

to a negligible response at regional level. Regional farm diversity, in size and intensity, represents diversity in management strategies (e.g., cultivar choice, and fertilizer and pesticide use). The opposite but small effect of land-use diversity suggests that increasing land-use diversity is not a good adaptation strategy for reducing the negative impacts of higher temperatures on regional wheat yields.

Our results give no information about the position and distance of the regions analyzed with respect to the thresholds beyond which structural system changes would occur. Farmers in Mediterranean regions may be closer to extreme weather conditions that make farming more unprofitable than in other regions. If temperatures become too high and/or water availability too low, even a high adaptive capacity may not allow farmers to cope anymore with "exposure" that has become too high. As was already stated by Odum (1969) for natural ecosystems, stresses can increase stability, but when stresses are too sudden or too violent, adaptation may not be fast enough, and stresses decrease rather than increase stability.

This study referred to wheat as is the most important crop in Europe, and it is grown in almost all regions. We have not considered other indicators of agricultural performance, but found similar results for other crops such as maize (Zea mays L. ssp. mays) and potato (Solanum tuberosum) with more negative effects of higher temperature in temperate compared with Mediterranean regions. The effects of climatic conditions on farmers' incomes are relatively small in Europe (Reidsma et al. 2007) as 
Fig. 4. Spatial distribution of the correlation between inter-annual variability in temperature and waterlimited yields [r(Ywat,temp)], and relationships to average temperature (temp, ${ }^{\circ} \mathrm{C}$ ) from 1990-2003. In contrast to what is observed in Fig. 2, the crop model projects negative impacts of higher temperatures, especially in regions with already higher temperatures.

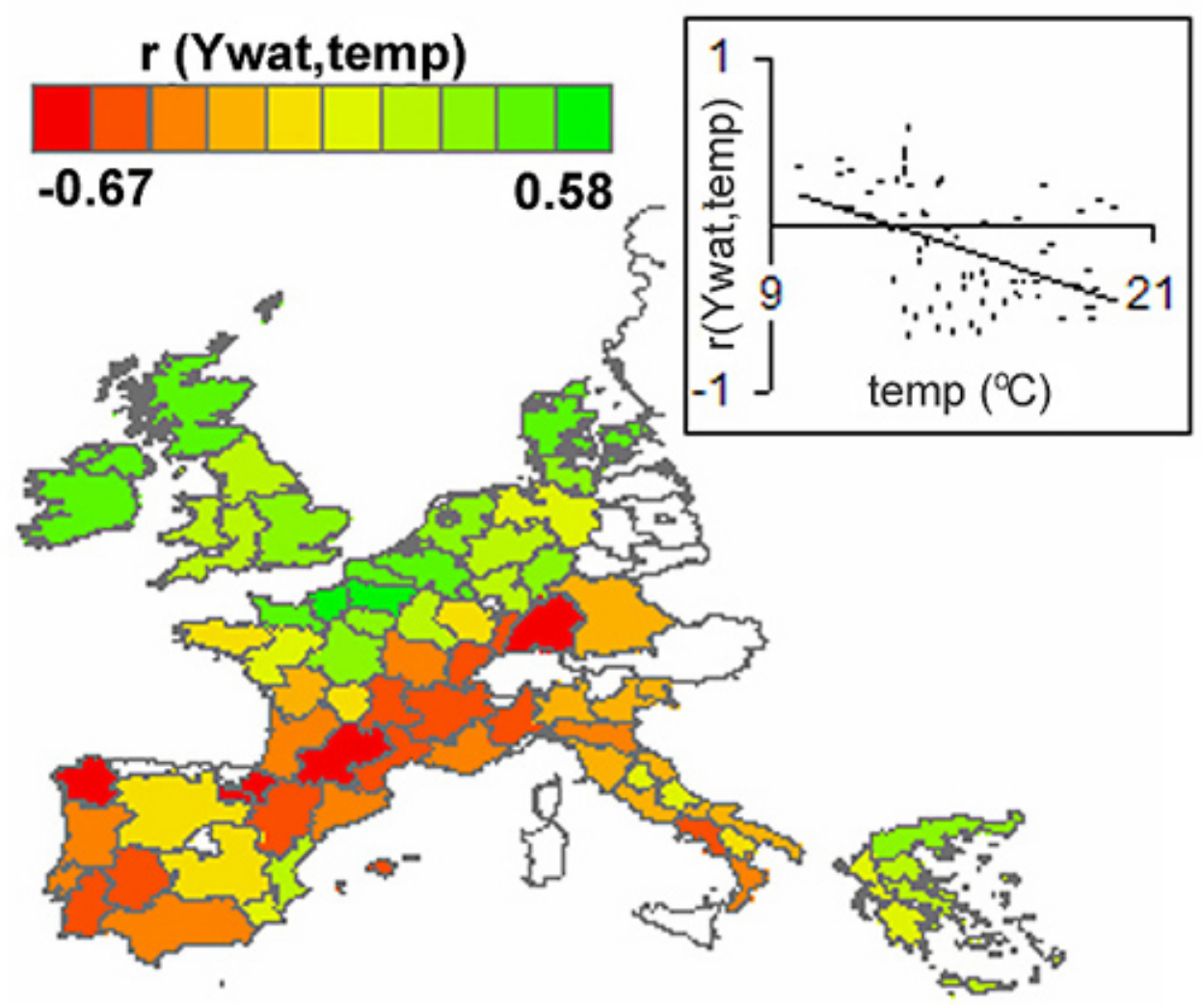

incomes largely depend on subsidies and market conditions. A more complex approach is thus needed to unravel the impacts on farmers' incomes specifically related to climatic variability and climate change. Although in general not significant, we observed especially negative effects of high temperatures on farmers' incomes in French regions with low regional farm diversity.

Diversity in management strategies is primarily determined by biophysical conditions, farming objectives, and perceptions. Explaining these relationships is not an aim of this analysis, but may be important for planning adaptation. It is not argued in this paper that the adaptation in terms of farm diversification as currently observed is based on conscious planned adaptation. A characteristic feature of complex adaptive systems is self- organization without intent (Levin 1998, Walker et al.2004). Although the dynamics of socioecological systems (here: agricultural systems) are dominated by human actors (here: farmers) who do exhibit intent, the system as a whole does not. Adaptation can be planned by institutions, but a region can only to a limited extent be considered as an actor with the ability to adapt. High farm diversity can be an emergent property in regions where farmers adapt their management in different ways.

Nevertheless, the observation that farm diversity can reduce the vulnerability of regional food production to climate variability points to a promising regional adaptation strategy for agriculture to climate change that has been largely overlooked so far. Implications for agriculture are considerable as present developments in many 
Fig. 5. Spatial distribution of the correlation between inter-annual variability in wheat yield anomalies and temp ${ }_{\text {month }}$ (the monthly temperature variable with the largest negative effect) [r(yield,temp month $_{\text {th }}$ ], and relationships to average temperature (temp, $\left.{ }^{\circ} \mathrm{C}\right)$ from $1990-2003$.

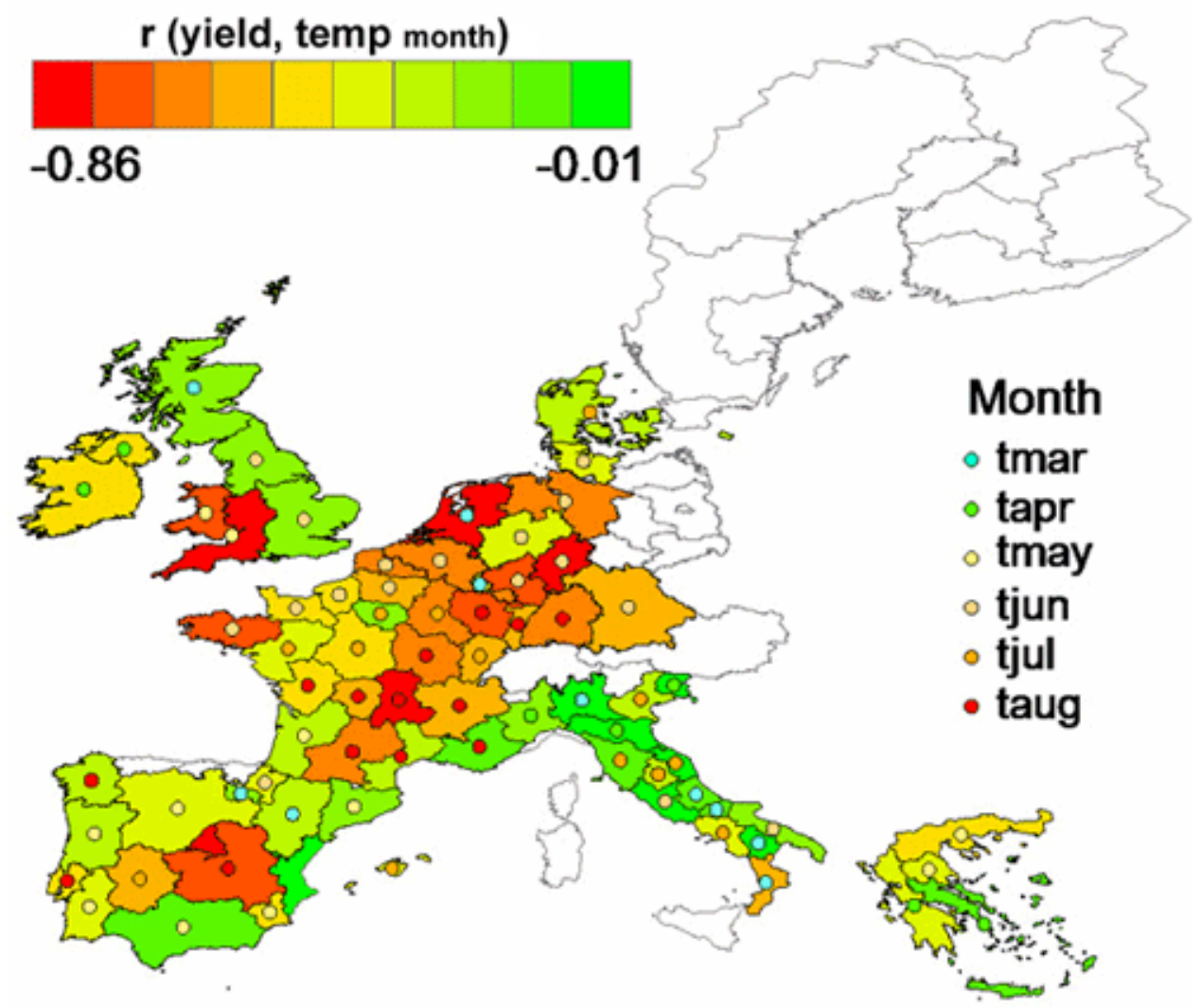

countries reduce farm diversity, which requires attention. Planned adaptation at higher aggregation levels is needed in order to cope with climate change and associated climate variability.

This has implications for subsidy, support, and incentive programs. Clearly, in the face of climate change, these measures should support the (increasing) diversity of farming systems rather than stimulating large, intensive, and specialized systems. Past measures of the Common Agricultural Policy (CAP) have mainly supported production and indirectly increased productivity. Recent reforms of the CAP toward a more sustainable agriculture may positively affect the diversity of farming objectives and management strategies in regions. Measures that will give farmers a choice (e.g., between irrigation or no irrigation, to use synthetic or organic fertilizers, between crop types and cultivars) will increase the diversity of farming systems and improve the adaptive capacity of a region. Importantly, the evaluation of such measures should consider an analysis of the temporal variability (stability) of the performance indicators.

How results of this study transfer to other regions needs to be assessed. Although the impact of regional farm diversity has not been assessed elsewhere, studies for Africa (Challinor et al. 2007) and Australia (Nelson et al. 2005) also showed that farms in less favorable regions are not necessarily more vulnerable to climate change. Farmers and agricultural regions have proved to be highly adaptable. As this study covers a large spatial scale, we anticipate that results are also valid for other regions. 
Fig. 6. (a) Spatial distribution of the variation in farm type yield variability (SD, \%), and relationships to average temperature (temp, ${ }^{\circ} \mathrm{C}$ ) from 1990-2003. Wheat yield variability is similar for different farm types in (b) Champagne-Ardenne, whereas in (c) Emilia-Romagna the diversity in wheat yield variability is larger. In (d) Champagne-Ardenne, standard deviations in the relative wheat yield anomaly for individual years are small $(\mathrm{SD}=3.7)$ and regional yield anomalies (from the trend) are significantly different from zero and correlated to temperature, $\left(r=-0.66\right.$ with temp $\mathrm{July}_{\mathrm{y}}, r=-0.44$ with temp). However, in (e) Emilia-Romagna, the standard deviations are large (SD $=8.3$ ) and regional yield anomalies are not significantly different from zero and are not significantly correlated to temperature $(r$ $=-0.13$ with temp April $_{1}, r=0.33$ with temp). Note, temperatures shown in (d) and (e) refer to the months with the largest negative correlation.
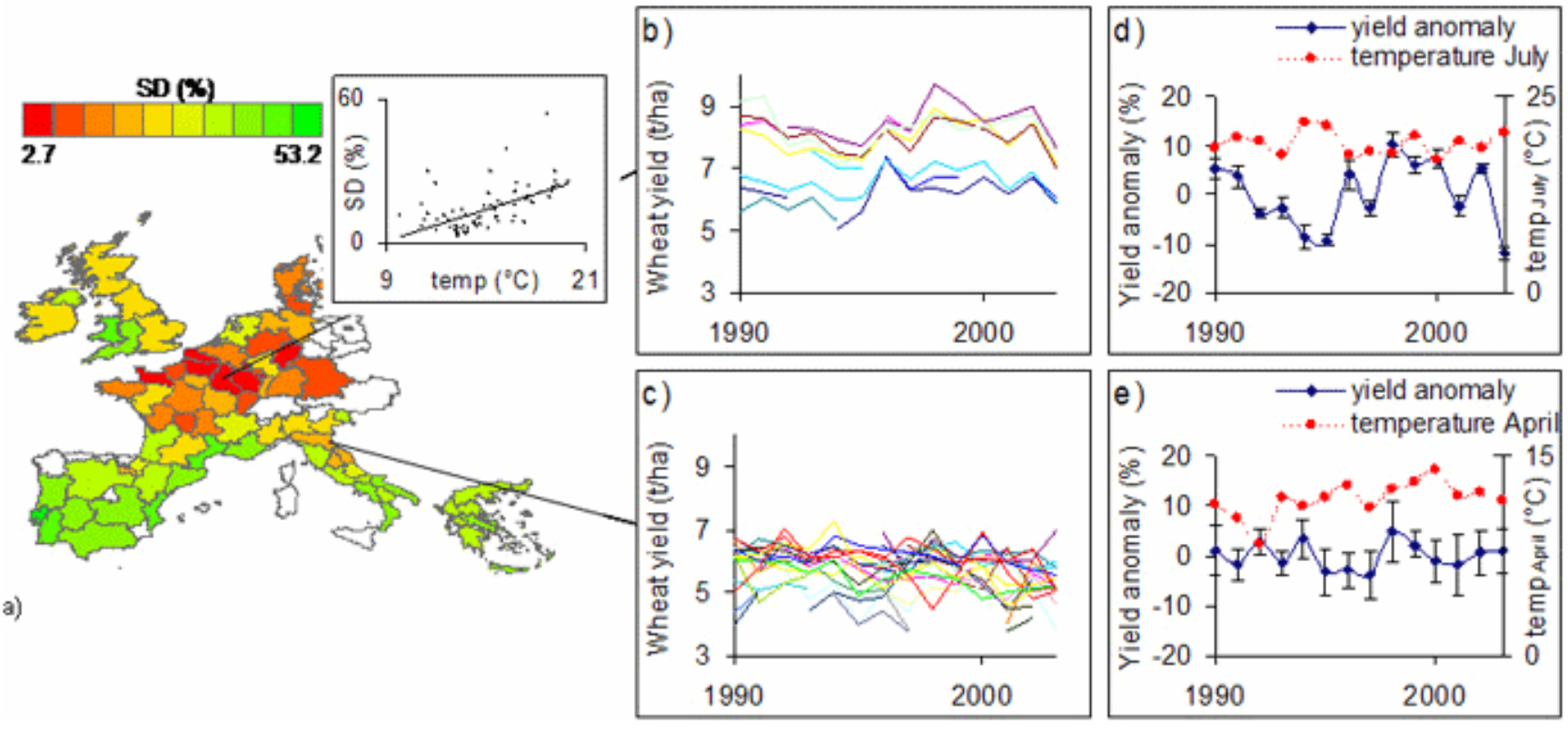

\section{The Empirical Analysis}

Climate effects on wheat yields are analyzed based on the Pearson correlation coefficient between climate variables and wheat yields. The Pearson correlation coefficient does not measure the extent of the impact but whether or not there is a relationship between yields and climate. It is a simple, straightforward, and appropriate measure for analyzing relationships between two variables. The stronger the relationship, the more yield variability can be attributed to climate variability. Yield variability not explained by climate variability, can be attributed to management (e.g., van Ittersum et al. 2003). The use of this simple measure can be debated, but it gives first insights into a complex subject.
Nevertheless, when analyzing complex systems, valid and reliable input data are required. We used climate variables that were aggregated from daily weather data. The validity of using these aggregated climate variables has been tested by comparing results with outputs from a crop simulation model. Crop simulation models use daily weather data to simulate yearly wheat yields. The results demonstrate that water-limited yields (wheat in Europe is generally not irrigated) are clearly negatively related to the aggregated temperature variable in Mediterranean regions, and more often positively in temperate regions; contrary to relationships found for actual yields in this study. So, the impact of higher average temperatures is different from what is expected based on biophysical relationships, compared with what is observed based on interactions between biophysical 
Table 2. Results from the regression model with r(yield, temp) as dependent variable and farm diversity and composition and prevailing climate conditions as independent variables (see text for further explanation).

\begin{tabular}{ll}
\hline \hline Variables & Estimates \\
\hline Intercept & -0.70 \\
Size diversity $\left(\mathrm{H}_{\mathrm{d}}{ }^{\prime}\right)$ & 0.91 \\
Intensity diversity $\left(\mathrm{H}_{\mathrm{d}}{ }^{\prime}\right)$ & 0.85 \\
Land-use diversity $\left(\mathrm{H}_{\mathrm{d}}{ }^{\prime}\right)$ & -0.49 \\
Medium intensive farm (fraction) & 0.46 \\
Small-scale farm (fraction) & 0.44 \\
Medium-scale farm (fraction) & -0.73 \\
$\mathrm{R}^{2}$ & 0.53 \\
\hline
\end{tabular}

relationships and management. Using these aggregated climate variables to measure climate effects and to determine the impact of management and adaptation is thus justified.

We acknowledge that there can be various reasons for finding differences in climate effects on wheat yields in Europe. Regional differences in, e.g., socioeconomic conditions or cultivars often exhibit a strong north-south gradient. However, the farm type yield variability (SD) as presented in Fig. 6 clearly demonstrates that there is a larger heterogeneity in the yield responses of different farm types in southern regions. If the main cause of the small climate effects would be related to growing different cultivars, this effect would be similar for all farm types and the SD would be smaller. The large SD demonstrates that diversity in management strategies is clearly important in reducing the responsiveness of a region to climate variability.

\section{CONCLUSION}

Temperature negatively affects regional wheat yields across Europe. However, effects of higher temperatures on wheat yields are smaller in Mediterranean regions compared with temperate regions. The diversity in farm size and intensity, particularly high in Mediterranean regions, reduces regional vulnerability of wheat yields to climate variability. Accordingly, farm diversification is a strategy through which regions in Europe can adapt to unfavorable conditions related to higher temperatures and associated droughts, which will increase with climate change.

Responses to this article can be read online at:

http://www.ecologyandsociety.org/voll3/iss 1/art38/responses/

\section{Acknowledgments:}

We thank the Netherlands Environmental Assessment Agency (MNP) for funding the PhD project of PR in which this research was carried out. We also thank the EU-funded SEAMLESS project (System for Environmental and Agricultural Modelling; Linking European Science and Society) for providing the FADN data and funding for FE. Furthermore, we thank the European Commission's Joint Research Centre (JRC) and Hendrik Boogaard for providing the climate data. Lastly, we gratefully acknowledge comments on earlier versions of this manuscript from Alfons Oude Lansink, Rik Leemans, Ken Giller, Mark Rounsevell, and two anonymous reviewers. 


\section{LITERATURE CITED}

Andersen, E., B. Elbersen, F. Godeschalk, and D. Verhoog. 2007. Farm management indicators and farm typologies as a basis for assessments in a changing policy environment. Journal of Environmental Management 82:353-362.

Andersen, E., A. D. Verhoog, B. S. Elbersen, F. E. Godeschalk, and B. Koole. 2006. A multidimensional farming system typology. ISBN 90-8585-041-X, SEAMLESS Report No.12, SEAMLESS integrated project, EU 6th Framework Programme [online] URL: www.seamless-ip.org/R eports/Report 12 PD4.4.2.pdf.

Bouwman, A. F., T. Kram, and K. Klein Goldewijk, editors. 2006. Integrated modelling of global environmental change. An overview of IMAGE 2.4 Netherlands Environmental Assessment Agency (MNP), Bilthoven, The Netherlands.

Calderini, D. F., and G. A. Slafer. 1998. Changes in yield and yield stability in wheat during the 20th century. Field Crops Research 57:335-347.

Challinor, A., T. Wheeler, C. Garforth, P. Craufurd, and A. Kassam. 2007. Assessing the vulnerability of food crop systems in Africa to climate change. Climatic Change 83:381-399.

Costanza, R., R. D'Arge, R. De Groot, S. Farber, M. Grasso, B. Hannon, K. Limburg, S. Naeem, R. V. O'Neill, J. Paruelo, R. G. Raskin, P. Sutton, and M. van Den Belt. 1997. The value of the world's ecosystem services and natural capital. Nature 387:253-260.

Díaz, S., and M. Cabido. 2001. Vive la différence: plant functional diversity matters to ecosystem processes. Trends in Ecology and Evolution 16:646655 .

Downing, T. E., R. E. Butterfield, S. J. Cohen, S. Huq, R. M. Moss, A. Rahman, Y. Sokona, and L. Stephen. 2001. Climate change vulnerability: linking impacts and adaptation. The Governing Council of the United Nations Environment Programme (UNEP), Nairobi, Kenya and Environmental Change Institute, University of Oxford, Oxford, UK.
Easterling, W. E., P. K. Aggarwal, P. Batima, K. M. Brander, L. Erda, S. M. Howden, A. Kirilenko, J. Morton, J.-F. Soussana, J. Schmidhuber, and F. N. Tubiello. 2007. Food, fibre and forest products. Pages 273-313 in Intergovernmental Panel on Climate Change (IPCC). Climate change 2007-impacts, adaptation and vulnerability. Working Group II Contribution to the Fourth Assessment Report of the IPCC. Cambridge University Press, Cambridge, UK.

Ellis, F. 2000. Rural livelihoods and diversity in developing countries. Oxford University Press, Oxford, UK.

Ewert, F., J. R. Porter, and M. D. A. Rounsevell. 2007. Crop models, $\mathrm{CO}_{2}$, and climate change. Science 315:459-460.

Ewert, F., M. D. A. Rounsevell, I. Reginster, M. J. Metzger, and R. Leemans. 2005. Future scenarios of European agricultural land use. I: Estimating changes in crop productivity. Agriculture, Ecosystems and Environment 107:101-116.

Fletcher, C. S., and D.W. Hilbert. 2007. Resilience in landscape exploitation systems. Ecological Modelling 201:440-452.

Fraser, E. D. G., W. Mabee, and F. Figge. 2005. A framework for assessing the vulnerability of food systems to future shocks. Futures 37:465-479.

Gitay, H., S. D. Brown, W. Easterling, and B. Jallow. 2001. Ecosystems and their goods and services. Pages 237-315 in Intergovernmental Panel on Climate Change (IPCC). Climate change 2001: impacts, adaptation and vulnerability. Working Group II Contribution to the Third Assessment Report of the IPCC. Cambridge University Press, Cambridge, UK.

Gunderson, L. H., and C. S. Holling. 2002. Panarchy: understanding transformations in human and natural systems. Island Press, Washington, D. C., USA.

Intergovernmental Panel on Climate Change (IPCC). 2001. Climate Change 2001: impacts, adaptation and vulnerability. Cambridge University Press, Cambridge, UK. 
2007. Climate change 2007-impacts, adaptation and vulnerability. Working Group II Contribution to the Fourth Assessment Report of the IPCC. Cambridge University Press, Cambridge, UK.

Lazar, C., and G. Genovese. 2004. Methodology of the MARS crop yield forecasting system. Volume 2. Agrometeorological modelling, processing and analysis. European Commission - Joint Research Centre, Brussels, Belgium. [online] URL: http://ma rs.jrc.it/marsstat/Crop\%5FYield\%5FForecasting/ METAMP/00000002.HTM.

Levin, S. A. 1998. Ecosystems and the biosphere as complex adaptive systems. Ecosystems 1:431-436.

Metzger, M. J., M. D. A. Rounsevell, L. AcostaMichlik, R. Leemans, and D. Schroter. 2006. The vulnerability of ecosystem services to land use change. Agriculture, Ecosystems and Environment 114:69-85.

Nelson, R., P. Kokic, L. Ellistion, and J. King. 2005. Structural adjustment: a vulnerability index for Australian broadacre agriculture. Australian Commodities 12:171-179.

Odum, E. P. 1969. The strategy of ecosystem development. Science 164:262-270.

Olesen, J. E., and M. Bindi. 2002. Consequences of climate change for European agricultural productivity, land use and policy. European Journal of Agronomy 16:239-262.

Parry, M. L., C. Rosenzweig, A. Iglesias, M. Livermore, and G. Fischer. 2004. Effects of climate change on global food production under SRES emissions and socio-economic scenarios. Global Environmental Change 14:53-67.

Reidsma, P. 2007. Adaptation to climate change: European agriculture. Dissertation, Wageningen University, Wageningen, The Netherlands. [online] URL: http://library.wur.nl/wda/dissertations/dis4314. pdf.

Reidsma, P., F. Ewert, and A. Oude Lansink. 2007. Analysis of farm performance in Europe under different climate and management conditions to improve understanding of adaptive capacity. Climatic Change 84:403-422.
Schar, C., P. L. Vidale, D. Luthi, C. Frei, C. Haberli, M. A. Liniger, and C. Appenzeller. 2004. The role of increasing temperature variability in European summer heatwaves. Nature 427:332-336.

Schröter, D., W. Cramer, R. Leemans, I. C. Prentice, M. B.Araújo, N. W.Arnell, A. Bondeau, H. Bugmann, T. R. Carter, C. A. Gracia, A. C. d. l. Vega-Leinert, M. Erhard, F. Ewert, M. Glendining, J. I. House, S. Kankaanpää, R. J. T. Klein, S. Lavorel, M. Lindner, M. J. Metzger, J. Meyer, T. D. Mitchell, I. Reginster, M. Rounsevell, S. Sabaté, S. Sitch, B. Smith, J. Smith, P. Smith, M. T. Sykes, K. Thonicke, W. Thuiller, G. Tuck, S. Zaehle, and B. Zierl. 2005. Ecosystem service supply and vulnerability to global change in Europe. Science 310:1333-1337.

Shannon, C. E., and W. Weaver. 1949. The mathematical theory of communication. The University of Illinois, Urbana, Chicago, Illinois, USA; London, UK.

Smit, B., O. V. Pilifosova, I. Burton, B. Challenger, S. Huq, R. J. T. Klein, G. Yohe, N. Adger, T. Downing, E. Harvey, S. Kane, M. L. Parry, M.Skinner, J.Smith, and J. Wandel. 2001. Adaptation to climate change in the context of sustainable development and equity. Pages 876912 in IPCC. Climate change 2001: impacts, adaptation and vulnerability. Cambridge University Press, Cambridge, UK.

Smit, B., and M. Skinner. 2002. Adaptation options in agriculture to climate change: a typology. Mitigation and Adaptation Strategies for Global Change 7:85-114.

Tilman, D., P. B. Reich, and J. M. H. Knops. 2006. Biodiversity and ecosystem stability in a decadelong grassland experiment. Nature 441:629-632.

- 2007. Ecology: diversity and stability in plant communities (Reply). Nature 446:E7-E8.

van der Dries, A. 2002. The art of irrigation. The development, stagnation and redesign of farmermanaged irrigation systems in northern Portugal. Dissertation, Wageningen University, Wageningen, The Netherlands.

van Ittersum, M.K., F. Ewert, T. Heckelei, J. Wery, J. Alkan Olsson, E. Andersen, I. Bezlepkina, S. Brogaard, M. Donatelli, G. 
Flichman, L. Olsson, A. Rizzoli, T. van der Wal, J. E. Wien, and J. Wolf. 2008. Integrated assessment of agricultural systems - a componentbased framework for the European Union (SEAMLESS). Agricultural Systems 96:150-165.

van Ittersum, M. K., P. A. Leffelaar, H. van Keulen, M. J. Kropff, L. Bastiaans, and J. Goudriaan. 2003. On approaches and applications of the Wageningen crop models. European Journal of Agronomy 18:201-234.

Walker, B., C. S. Holling, S. R. Carpenter, and A. P. Kinzig. 2004. Resilience, adaptability and transformability in social-ecological systems. Ecology and Society 9(2): 5. [online] URL: http://w ww.ecologyandsociety.org/vol9/iss2/art5. 